\title{
Pathological and Immunohistochemical Studies of Experimental Mycoplasma pneumoniae in Gerbils (Meriones unguiculatus)
}

\author{
Francisco Rodríguez, Ana S Ramírez, Pedro Castro and José B Poveda \\ Institute for Animal Health, Veterinary School, Universidad de Las Palmas de Gran Canaria, Arucas, Gran Canaria, Spain
}

\begin{abstract}
Summary
Mycoplasma pneumoniae (Mp) is a leading cause of human community-acquired pneumonia. To investigate the pathogenesis of the infection, 36 gerbils were intranasally inoculated with Mp culture (30 animals) or sterile mycoplasma broth (6 animals) and euthanized from 1 to 5 weeks post infection. A morphological and immunohistochemical study was carried out in all animals to determine the cellular populations present in lung parenchyma. Polyclonal and monoclonal antibodies were used to detect antigens of Mp and CD3, CD4, CD8 and CD79 lymphocytes, as well as cells containing S100 and major histocompatibility complex class II (MHC-II) antigens. There was progressive infiltration of mononuclear cells in the lamina propria of bronchi and bronchioles, and hyperplasia of the bronchus-associated lymphoid tissue (BALT) in the infected animals. BALT contained dendritic cells immunopositive to S100 and MHC-II and numerous CD3, CD4 and CD79 lymphocytes. The immunohistochemical results showed that T lymphocytes, particularly CD4 and CD79 cells, may play a role in the immune response of gerbils against $\mathrm{Mp}$. This experimental model is valuable for investigation of the pathogenesis of $\mathrm{Mp}$ infection and may assist in the development of therapeutic strategies.
\end{abstract}

(c) 2021 Elsevier Ltd. All rights reserved.

Keywords: bronchus-associated lymphoid tissue; gerbil; immunohistochemistry; Mycoplasma pneumoniae

\section{Introduction}

Mycoplasma pneumoniae $(\mathrm{Mp})$ is one of the main causes of atypical pneumonia, and is associated with pharyngitis, bronchitis, bronchiolitis, bronchiolitis obliterans, pericarditis, meningoencephalitis and arthritis (Neumayr et al, 2003; Yáñez et al, 2013). However, the pathogenesis of the disease is not completely clear. It seems to be primarily caused by adsorption of $\mathrm{Mp}$ to the respiratory epithelium and direct invasion of these cells, followed by a host immune response (Moore et al, 2014). Although epithelial cells seem to play an important role in recruiting inflammatory cells into the airways, the pathogenic mechanisms for lung inflammation have not been well-defined (Dakhama et al, 2003; Waites and Talkington, 2004).

Correspondence to: F Rodríguez (e-mail: francisco.guisado@ulpgc.es).
Mp, a common pathogen of community-acquired pneumonia in humans, usually follows a benign course, but occasionally the infection can develop into severe life-threatening conditions, including refractory Mp pneumonia, acute respiratory distress syndrome, necrotizing pneumonitis and fulminant pneumonia (Wang et al, 2004). The most characteristic pathological feature of Mp pneumonia in humans is marked plasma cell-rich lymphocytic infiltration in the peribronchovascular areas (Rollins et al, 1986; Saraya et al, 2014). As the disease progresses, the changes consist of the presence of mononuclear cells around bronchi and bronchioles and prominent lymphoid hyperplasia of the bronchus-associated lymphoid tissue (BALT).

Animal models of $\mathrm{Mp}$ pneumonia include experimental infections in mice (Fonseca-Aten et al, 2005; Tagliabue et al, 2008; Sekine et al, 2009; Saraya et al, 
2014) and hamsters (Loveless et al, 1992; Yáñez et al, 2013). However, animal models have not been able to fully reproduce the pathological features of human Mp pneumonia, especially the persistent infiltration of mononuclear cells in peribronchiolar spaces (Saraya et al, 2011).

We investigated the use of gerbils as an animal model for the induction of experimental pneumonia by intranasal inoculation of Mp. Pathological and immunological examinations were performed to examine the immunoinflammatory mechanisms involved in the disease and to compare them with previous studies. The model could provide data for evaluating factors involved in microorganism colonization, immunopathogenic mechanisms and treatment regimens.

\section{Materials and Methods}

\section{Experimental Design}

Tissues were obtained from 36 Mongolian gerbils (Meriones unguiculatus) (Strain 243; Charles River Laboratory International, Wilmington, Massachussetts, USA). Thirty animals were inoculated intranasally at 12 weeks of age (45-73 g body weight) with $10^{8}$ colony-forming units/ml of Mp (ATCG 29342, LGC Standards SLU, Barcelona, Spain) diluted in $100 \mu \mathrm{l}$ of mycoplasma broth. Mp cells were cultured at $37^{\circ} \mathrm{C}$ under a $5 \% \mathrm{CO}_{2}$ atmosphere for 4 days in Friis broth. Six inoculated animals (three males and three females) were killed at each of weeks 1 to 5 post infection (wpi) (Table 1). The remaining six gerbils were used as negative controls and killed in pairs at 1,3 and 5 weeks after inoculation, by the same route, with sterile Friis culture medium. Euthanasia was carried out by exsanguination under sedation induced by intramuscular injection of $0.2 \mathrm{~g} / \mathrm{kg}$ dexmedetomidine hydrochloride. The study was performed in strict accordance with the recommendations of the Guide for the Care and Use of Laboratory Animals (Directive, 2010/63/UE) and the protocol was reviewed and approved by the Ethics Committee for Animal Experimentation of the University of Las Palmas de Gran Canaria (EAECULPGG) (Ref. 006/2014).

\section{Pathology}

At necropsy, lung tissues were removed and processed for histological examination and immunohistochemistry. Tissues were fixed in 10\% neutral-buffered formalin, embedded in paraffin wax, sectioned $(4 \mu \mathrm{m})$ and stained with haematoxylin and eosin (HE). The histopathological lung score (HPL) was determined by grading the severity of peribronchiolar and peribronchial infiltrates, bronchiolar and bronchial luminal exudate, perivascular infiltrates and parenchymal pneumonia, as described (Cimolai et al, 1992). Using this system, the severity of lung lesions was evaluated from 0 to 26 with higher numbers indicating more severe lesions.

\section{Immunohistochemistry}

Tissue sections were dewaxed and rehydrated, and endogenous peroxidase activity was blocked by incubation with $\mathrm{H}_{2} \mathrm{O}_{2} \quad 0.3 \%$ in methanol for $30 \mathrm{~min}$ at room temperature. The sections were then subjected to heat-induced antigen retrieval (water bath at $98^{\circ} \mathrm{C}$ ) with an antigen retrieval solution, $\mathrm{pH} 6.0$ (Dako, Glostrup, Denmark) for $10 \mathrm{~min}$. All tissue sections were incubated with $10 \%$ goat normal serum (Vector Laboratories, Burlingame, California, USA) for $30 \mathrm{~min}$ at room temperature. The primary reagents, diluted 1:500, which included mouse monoclonal (CD4, CD8, CD79 and MHC-II) and rabbit polyclonal (S100 and CD3) antibodies (Thermo Fisher Scientific, Madrid, Spain), were then applied overnight at $4^{\circ} \mathrm{C}$. Lymph node sections from control

Table 1

Immunohistochemical findings in Mycoplasma pneumoniae-infected and control gerbils

\begin{tabular}{|c|c|c|c|c|c|c|}
\hline \multirow[t]{2}{*}{ Antibody } & \multirow[t]{2}{*}{ Controls } & \multicolumn{5}{|c|}{ Weeks post infection } \\
\hline & & 1 & 2 & 3 & 4 & 5 \\
\hline $\mathrm{S} 100 *$ & $2.1 \pm 0.9$ & $10.3 \pm 1.3$ & $13.3 \pm 2.4$ & $12.4 \pm 0.9$ & $12.5 \pm 1.6$ & $13.6 \pm 2.2$ \\
\hline $\mathrm{CD} 3 *$ & $4.2 \pm 1.5$ & $14.1 \pm 3.4$ & $15.3 \pm 0.9$ & $14.8 \pm 3.6$ & $16.3 \pm 3.3$ & $17.5 \pm 2.6$ \\
\hline $\mathrm{CD} 4 *$ & $3.7 \pm 0.9$ & $11.8 \pm 3.4$ & $12.4 \pm 2.9$ & $15.9 \pm 2.6$ & $11.3 \pm 2.7$ & $13.5 \pm 1.3$ \\
\hline CD8 & $4.1 \pm 1.8$ & $6.3 \pm 4.6$ & $5.5 \pm 3.8$ & $4.5 \pm 2.7$ & $5.3 \pm 1.3$ & $6.8 \pm 3.9$ \\
\hline CD79* & $1.2 \pm 3.9$ & $16.3 \pm 4.7$ & $13.5 \pm 3.3$ & $12.5 \pm 1.6$ & $14.7 \pm 2.4$ & $12.3 \pm 2.2$ \\
\hline MHC-II* & $3.8 \pm 2.7$ & $9.3 \pm 1.4$ & $16.4 \pm 2.2$ & $15.5 \pm 2.9$ & $12.3 \pm 3.3$ & $18.5 \pm 3.1$ \\
\hline
\end{tabular}

Results expressed as mean number of immunolabelled cells $/ \mathrm{mm}^{2} \pm \mathrm{SD}$.

*Significant differences $(P \leq 0.05)$ between infected and control animals. 
gerbils were run alongside the immunohistochemical procedures to check the cross-reactivity of all primary antibodies with gerbil tissues.

A biotinylated rabbit anti-mouse or swine antirabbit IgG (Vector Laboratories), diluted 1:200, was applied as secondary reagent for $30 \mathrm{~min}$ at room temperature. An avidin-biotin complex (Vectastain; Vector Laboratories), diluted 1:50, was applied as the third reagent. The sections were incubated for 3 min with 3,3'-diaminobenzidine tetrahydrocloride (Sigma, St. Louis, Missouri, USA) $0.035 \%$ in Tris-buffered saline (TBS) containing $0.1 \% \mathrm{H}_{2} \mathrm{O}_{2}$. After rinsing in tap water, the slides were lightly counterstained with Harris's haematoxylin and mounted under DPX mountant (BDH Laboratory Supplies, Poole, England) for microscopy. Mp antigen was immunolabelled as previously described (Sarradell et al, 2003) by using a monoclonal antibody (Biosource, California, USA) diluted 1:1000. Sections in TBS, or treated with normal goat serum or inappropriate antibodies (rabbit anti-swine IgG or mouse anti-goat $\operatorname{IgG})$ to replace the primary antibodies, were included as negative controls.

\section{Statistical Analysis}

Immunolabelled cells were counted at $\times 400$ magnification in 20 randomly selected fields from each animal and the results expressed as the mean \pm standard deviation (SD) of positive cells/ $\mathrm{mm}^{2}$ for each group of animals. A comparative analysis of immunolabelled cells in the control and infected animals at each time point was made by using the non-parametric Mann-Whitney $U$ test or the Kruskal-Wallis test by means of the SPSS v.26 computer program (SPSS, Chicago, Illinois, USA). $P \leq 0.05$ was considered statistically significant.

\section{Results}

\section{Pathology}

Macroscopically, multiple pale nodules, $<1 \mathrm{~mm}$ in diameter, indicative of lymphoid hyperplasia, were observed against a red background in the lungs of infected gerbils. Histologically, these areas had lesions of bronchointerstitial pneumonia (Fig. 1) with prominent lymphoreticular hyperplasia of BALT and thickening of alveolar septa by mononuclear cell infiltrates. The severity of inflammation correlated with the course of the disease (Fig. 2). Mp was reisolated and detected by real-time polymerase chain reaction targeting gene $P 1$ in all infected animals, as described (Kerdsin et al, 2010; Yáñez et al, 2013). The involvement of other respiratory pathogens was excluded by immunohistological and microbiological techniques, which included incubation of lung samples in Hayflick and SP4 media for mycoplasma isolation, as well as aerobic and anaerobic cultures on blood

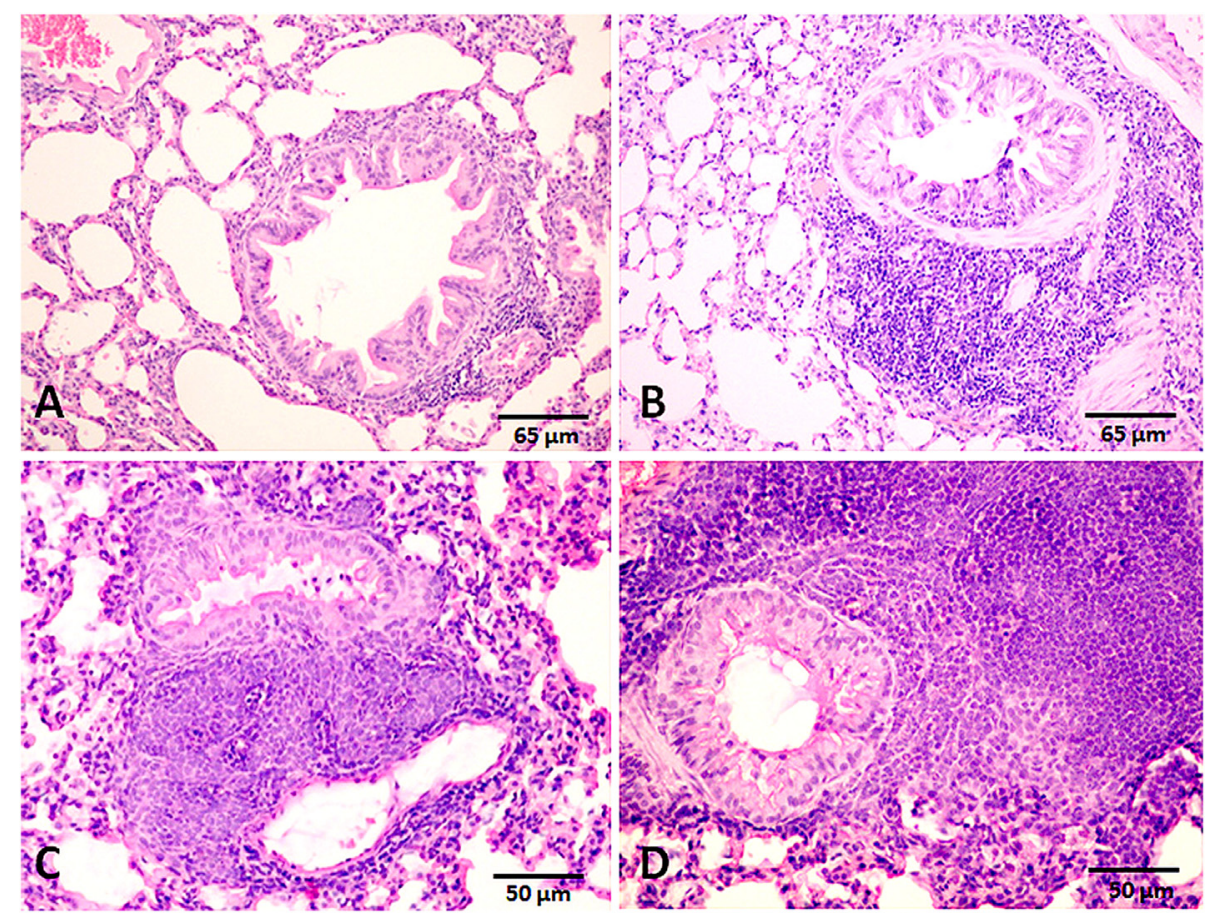

Fig. 1. Gerbil, lung, M. pneumoniae infection. Progressive increase in severity of peribronchiolar lymphoid hyperplasia and thickening of alveolar septa at 1 (A), 2 (B), 4 (C) and 5 (D) wpi. HE. 


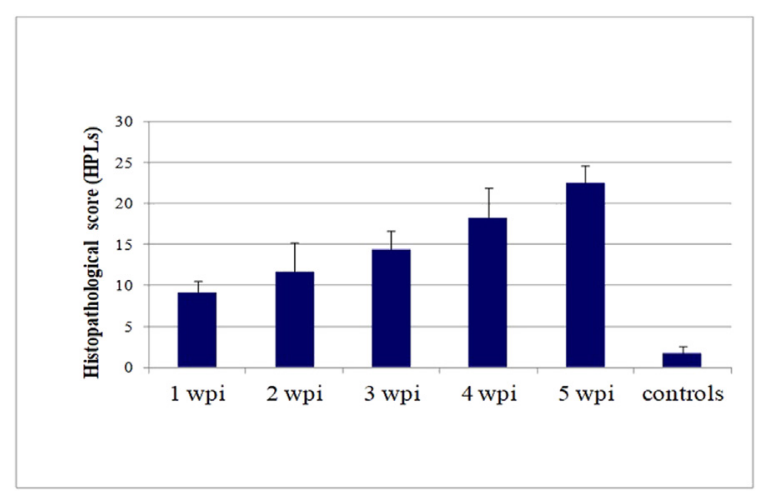

Fig. 2. Microscopic lung lesions scored as median HPLs \pm SD of control $(\mathrm{n}=6)$ and infected $(\mathrm{n}=30)$ gerbils with M. pneumoniae and euthanized 1 to 5 wpi. Significant differences $(P \leq 0.05)$ between infected and control animals at all time points.

agar and McConkey agar and in thioglycollate liquid medium.

\section{Immunohistochemical Labelling}

Mycoplasmal antigen was not detected in the uninfected control gerbils. Immunolabelling of Mp antigen was visualized as a brown granular reaction on the surface of bronchial and bronchiolar (Fig. 3A) epithelial cells of lung tissues from all infected animals.

The immunophenotypes of various cell subsets are shown in Table 1 and Figs. 3B-F. Diffuse nuclear and cytoplasmic immunolabelling with the S100 antibody was detected in stellate dendritic cells with long cytoplasmic processes that were mainly located in the germinal centres of BALT (Fig. 3B). Some lymphocytes, smooth muscle cells, nerve endings, chondrocytes and macrophages of the alveolar walls were also immunopositive. MHC-II antigen (Fig. 3C) was seen in lymphocytes and in cells with an irregular morphology and cytoplasmic prolongations, present in the central areas of BALT, between lymphocytes and under the epithelial basal membrane. CD3, CD4 and CD8 immunoreactivity was detected in the cytoplasm of lymphocytes in aggregates in germinal centres, scattered in perifollicular areas and infiltrating the epithelium and the lamina propria of bronchi and bronchioles (Figs. 3D-F). The number of CD4 lymphocytes was higher than that of CD8 cells in all infected gerbils, compared with control animals. Immunolabelling with CD79 antibody was found in the cytoplasm of many B lymphocytes in the follicular areas, in the lamina propria of bronchi and bronchioles, and within the airway epithelium.

\section{Discussion}

$\mathrm{Mp}$ is an extracellular pathogen that attaches to the bronchial and bronchiolar epithelial cells via adhesins on the bacterial cell surface (Dallo et al, 1988; Waites and Talkington, 2004), where it produces $\mathrm{H}_{2} \mathrm{O}_{2}$ and superoxide radicals, which induce oxidative stress in the respiratory epithelium. The aim of this study was to reproduce the inflammatory processes seen in human Mp pneumonia using an experimental model based on the induction of lesions in gerbils by intranasal inoculation of $\mathrm{Mp}$.

A common feature of mycoplasmal pneumonia is the persistence of the causal microorganisms at the mucosal surface and an increase in the number of mononuclear cells in the lung. These changes lead to peribronchiolar accumulations of these cells, many of which are likely to be synthesizing antibody as part of the immune response against the infection (Cassell et al, 1974). Several mycoplasmal products and mycoplasma membrane proteins, which are immunogenic, have been reported as responsible for the intense lymphoid hyperplasia around airways (Ross and Young, 1993; Saraya et al, 2014). BALT is composed of lymphoid follicles covered by a layer of specialized cuboidal epithelial cells without cilia, and participates in mucosal defence by trapping the antigens of inhaled particles (Bienestock, 1984; Sarradell et al, 2003).

In addition to producing a variety of inflammatory and immunoregulatory cytokines, macrophages and related cells of the myeloid lineage differentiate into antigen-presenting cells (APCs), known as dendritic cells because of their characteristic morphology (Bienestock, 1984; Murtaugh and Foss, 2002). APCs phagocytize, process and present antigen, which constitutes a crucial step in the induction of antigenspecific immunity at mucosal surfaces. In the current study, MHC-II and S100 immunopositive stellate cells with long cytoplasmic processes, characteristic of dendritic cells, were detected in the germinal centres of BALT. This observation indicates that this lymphoid tissue possesses a high structural organization in gerbils, which may facilitate presentation of antigen to lymphocytes. Understanding activation of this cell type in the mucosal environment is important to prevent infection at mucosal surfaces (Bienestock, 1984; Sarradell et al, 2003) because of the difficulties in inducing protective immunity at such sites through vaccination.

The severity of lesions produced under experimental conditions may be influenced by the degree of colonization or virulence of the mycoplasma strain used. In the conditions of the present experiment, $\mathrm{Mp}$ did not induce respiratory disease severe enough to 


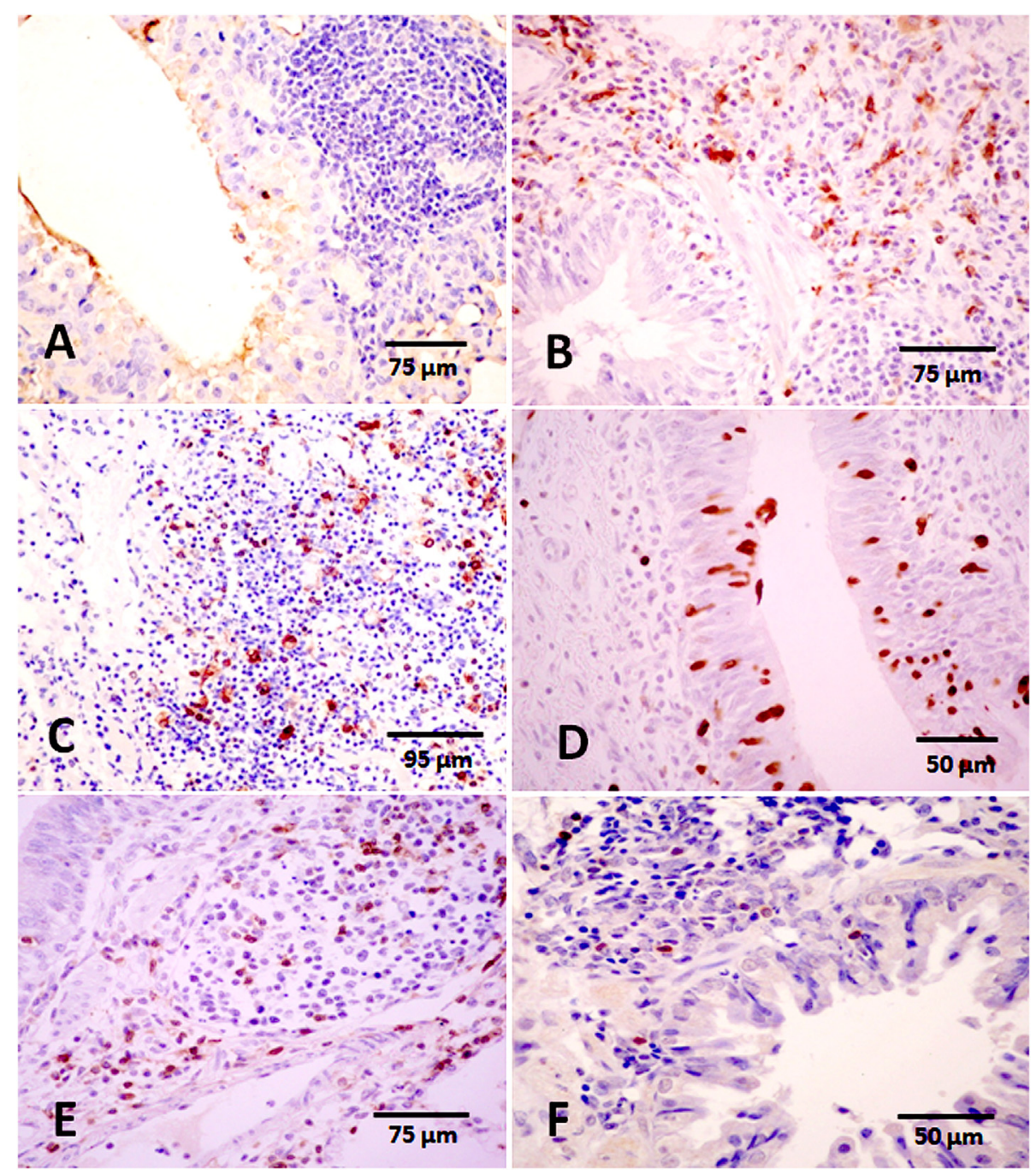

Fig. 3. Gerbil, lung, M. pneumoniae infection. (A) M. pneumoniae antigen in bronchiolar epithelium at 2 wpi. IHC. (B) Immunolabelling of $\mathrm{S} 100$ antigen in dendritic cells in germinal centres of BALT at 4 wpi. IHC. (C) Immunolabelling of MHC-II antigen in cytoplasm of mononuclear cells at 2 wpi. IHC. (D) Many CD3-positive lymphocytes between epithelial cells and in lamina propria at 4 wpi. IHC. (E) Immunolabelling of numerous CD4 lymphocytes in BALT at 5 wpi. IHC. (F) Scattered CD8 lymphocytes in lamina propria and BALT at 5 wpi. IHC.

cause death of any gerbil. Several studies have demonstrated that patients with humoural antibody deficiency seem to become chronic carriers or to undergo repeated episodes of $\mathrm{Mp}$ pneumonia (Roifman et al, 1986), indicating that humoral immunity plays a role in protection against these organisms. Furthermore, cellular immunity, with increased numbers of CD3-positive cells and with a predominance of CD4 over CD8 cells, as observed in this study, is related to the results obtained in bronchoalveolar lavage fluid of humans and experimentally infected animals (Saraya et al, 2014). This finding, probably due to non-specific reactions to $\mathrm{Mp}$ antigens, has been associated with the development of lung inflammation (Foy et al, 1973; Dakhama et al,
2003). Consequently, an excessive immune response, rather than direct microbial damage, is thought to be related to the severity of Mp pneumonia (Jones et al, 2002; Waites and Talkington, 2004; FonsecaAten et al, 2005). Furthermore, since CD8 lymphocytes have been shown to be important in the clearance of different infections from the respiratory tract (Moore et al, 2001; Dobbs et al, 2009; Schmidt and Varga, 2018), their relatively lower number compared with CD4 cells may be involved in the persistence of $\mathrm{Mp}$.

In the current study, Mp immunoreactivity on the surface of epithelial cells of the respiratory tract was associated with progressive infiltration of macrophages and lymphocytes in the peribronchiolar areas. 
In studies, carried out in cultures of human airway epithelium infected with Mp (Prince et al, 2018), apical surface infection was followed by pericellular invasion and colonization of the basolateral compartment. In contrast, $\mathrm{Mp}$ antigen was detected on the luminal surface of bronchial and bronchiolar epithelial cells, but not within epithelial cells, BALT or alveolar macrophages, in the current animal model. The increase in the severity of lung lesions in the advanced stages of the disease, as indicated by the intensity of lymphoid cell infiltration, supports the importance of the immunological reaction, possibly through the production of proinflammatory cytokines, which can activate macrophages, and $\mathrm{B}$ and $\mathrm{T}$ lymphocytes (Bienestock, 1984; Dakhama et al, 2003; Tagliabue et al, 2008), in addition to the direct action of the pathogen.

Studies in mice and humans have revealed that $\mathrm{Mp}$ causes persistent infection in the lower respiratory tract, which may upregulate host immunity (Hardy et al, 2002; Saraya et al, 2011). The efficacy of clarithromycin and dexamethasone therapy in reducing Mp-associated pneumonia (Tagliabue et al, 2008) and the response to immunomodulators in human patients suggest that lung injury in Mp infection is associated with a hyperactive host immune response rather than to the direct effects of $\mathrm{Mp}$ on respiratory cells.

In summary, this study demonstrates the ability of $\mathrm{Mp}$ to induce respiratory tract infection, to persist for several weeks at the mucosal surface and to cause progressive pneumonic lesions in the respiratory tract of experimentally inoculated gerbils. An excessive immune response, represented by macrophages and lymphocytes expressing S100, MHC-II, CD3, CD4 and CD79 antigens, seems to be the major contributing factor to the pathogenesis of $\mathrm{Mp}$ infection.

\section{Conflict of Interest Statement}

The authors declared no potential conflicts of interest with respect to the research, authorship or publication of this article.

\section{Acknowledgments}

The research was funded through our institute's own resources.

\section{References}

Bienestock J (1984) Bronchus-associated lymphoid tissue. In: Immunology of the Lung and Upper Respiratory Tract, J Bienenstock, Ed, McGraw-Hill, New York, pp 96-118.
Cassell GH, Lindsey JR, Baker HJ (1974) Immune response of pathogen-free mice inoculated intranasally with Mycoplasma pulmonis. The Journal of Immunology, 112, 124-136.

Cimolai N, Taylor GP, Mah D, Morrison BJ (1992) Definition and application of a histopathological scoring scheme for an animal model of acute Mycoplasma pneumoniae pulmonary infection. Microbiology and Immunology, 36, 465-478.

Dakhama A, Kraft M, Martin RJ, Gelfand EW (2003) Induction of regulated upon activation, normal T cells expressed and secreted (RANTES) and transforming growth factor-betalin airway epithelial cells by $M y c o-$ plasma pneumoniae. American Journal of Respiratory Cell and Molecular Biology, 29, 344-351.

Dallo SF, Su CJ, Horton JR, Baseman JB (1988) Identification of $P 1$ gene domain containing epitope(s) mediating Mycoplasma pneumoniae cytoadherence. Fournal of Experimental Medicine, 167, 718-723.

Dobbs NA, Odeh AN, Sun X, Simecka JW (2009) The multifaceted role of $\mathrm{T}$ cell-mediated immunity in pathogenesis and resistance to mycoplasma respiratory disease. Current Trends in Immunology, 10, 1-19.

Fonseca-Aten M, Rios AM, Mejias A, Chavez-Bueno S, Katz K et al (2005) Mycoplasma pneumoniae induces host-dependent pulmonary inflammation and airway obstruction in mice. American Gournal of Respiratory Cell and Molecular Biology, 32, 201-210.

Foy HM, Ochs H, Davis SD, Kenny GE, Luce RR (1973) Mycoplasma pneumoniae infections in patients with immunodeficiency syndromes: report of four cases. The fournal of Infectious Diseases, 127, 388-393.

Hardy RD, Jafri HS, Olsen K, Hatfield J, Iglehart J et al (2002) Mycoplasma pneumoniae induces chronic respiratory infection, airway hyperreactivity, and pulmonary inflammation: a murine model of infection-associated chronic reactive airway disease. Infection and Immunity, 70, 649-654.

Jones HP, Tabor L, Sun X, Woolard MD, Simecka JW (2002) Depletion of CD8-T cells exacerbates CD4-Th cell-associated inflammatory lesions during murine mycoplasma respiratory disease. The fournal of Immunology, 168, 3493-3501.

Kerdsin A, Uchida R, Verathamjamrus C, Puangpatra P, Kawakami K et al (2010) Development of triplex SYBR green real-time PCR for detecting Mycoplasma pneumoniae, Chlamydophila pneumoniae, and Legionella spp. without extraction of DNA. Japanese Fournal of Infectious Diseases, 63, 173-180.

Loveless RW, Griffiths S, Fryer PR, Blauth C, Feizi T (1992) Immunoelectron microscopic studies reveal differences in distribution of sialo-oligosaccharide receptors for Mycoplasma pneumoniae on the epithelium of human and hamster bronchi. Infection and Immunity, 60, 4015-4023.

Moore BB, Moore TA, Toews GB (2001) Role of T- and Blymphocytes in pulmonary host defences. European Respiratory Fournal, 18, 846-856. 
Moore C, Perry M, Cottrell S (2014) The emerging role of community sentinel surveillance in the understanding of the clinical features and epidemiology of acute $M y c o-$ plasma pneumoniae infection. Clinical Microbiology and Infections, 20, 489-492.

Murtaugh MP, Foss DL (2002) Inflammatory cytokines and antigen presenting cell activation. Veterinary Immunology and Immunopathology, 87, 109-121.

Neumayr L, Lennette E, Kelly D, Earles A, Embury S et al (2003) Mycoplasma disease and acute chest syndrome in sickle cell disease. Pediatrics, 112, 87-95.

Prince OA, Krunkosky TM, Sheppard ES, Krause DC (2018) Modelling persistent Mycoplasma pneumoniae infection of human airway epithelium. Cellular Microbiology, 20, e12810.

Roifman CM, Rao CP, Lederman HM, Lavi S, Quinn P et al (1986) Increased susceptibility to mycoplasma infection in patients with hypogammaglobulinemia. The American Fournal of Medicine, 80, 590-594.

Rollins S, Colby T, Clayton F (1986) Open lung biopsy in Mycoplasma pneumoniae pneumonia. Archives of Pathology ES Laboratory Medicine, 110, 34-41.

Ross RF, Young TF (1993) The nature and detection of mycoplasmal immunogens. Veterinary Microbiology, 37, 369-380.

Saraya T, Kurai D, Nakagaki K, Sasaki Y, Niwa S et al (2014) Novel aspects on the pathogenesis of Mycoplasma pneumoniae pneumonia and therapeutic implications. Frontiers in Microbiology, 5, 1-18.

Saraya T, Nakata K, Nakagaki K, Motoi N, Iihara K et al (2011) Identification of a mechanism for lung inflammation caused by Mycoplasma pneumoniae using a novel mouse model. Results in Immunology, 1, 76-87.

Sarradell J, Andrada M, Ramírez AS, Fernández A, Gómez-Villamandos JC et al (2003) A morphologic and immunohistochemical study of the bronchusassociated lymphoid tissue of pigs naturally infected with Mycoplasma hyopneumoniae. Veterinary Pathology, 40, 395-404.

Schmidt ME, Varga SM (2018) The CD8 T cell response to respiratory virus infections. Frontiers in Immunology, 9, 678.

Sekine H, Taguchi H, Watanabe H, Kawai S, Fujioka Y et al (2009) Immunological analysis and pathological examination of gnotobiotic mice mono-associated with Mycoplasma pneumoniae. Journal of Medical Microbiology, 58, 697-705.

Tagliabue C, Salvatore CM, Techasaensiri C, Mejias A, Torres JP et al (2008) The impact of steroids given with macrolide therapy on experimental Mycoplasma pneumoniae respiratory infection. The Journal of Infectious Diseases, 198, 1180-1188.

Waites KB, Talkington DF (2004) Mycoplasma pneumoniae and its role as a human pathogen. Clinical Microbiology Reviews, 17, 697-728.

Wang RS, Wang SY, Hsieh KS, Chiou YH, Huang IF et al (2004) Necrotizing pneumonitis caused by Mycoplasma pneumoniae in pediatric patients. Pediatric Infectious Disease, 23, 564-567.

Yáñez A, Martínez-Ramos A, Calixto T, GonzálezMatus FJ, Rivera-Tapia JA et al (2013) Animal model of Mycoplasma fermentans respiratory infection. BMC Research Notes, 6, 1-9.

$\left[\begin{array}{l}\text { Received, September 23rd, } 2020 \\ \text { Accepted, January 27th, } 2021\end{array}\right]$ 